Available at

www.ElsevierComputerScience.com

POWERED BY S C IENCE d DIRECT

\title{
Fuzzy location problems on networks
}

\author{
José A. Moreno Pérez ${ }^{\mathrm{a}, *}$, J. Marcos Moreno Vega ${ }^{\mathrm{a}}$, José L. Verdegay ${ }^{\mathrm{b}}$ \\ ${ }^{a}$ Dpto. Estadística, I.O. y Computación, Facultad de Matemáticas, Universidad de La Laguna, 38271 , \\ La Laguna, Spain \\ ${ }^{\mathrm{b}}$ Dpto. de Ciencias de la Computación e Inteligencia Artificial, E.T.S. de Ingeniería Informática, \\ Universidad de Granada, 18071, Granada, Spain
}

Received 20 September 2001; received in revised form 29 January 2003; accepted 25 February 2003

\begin{abstract}
Location problems concern a wide set of fields where it is usually assumed that exact data are known. However, in real applications, the location of the facility considered can be full of linguistic vagueness, that can be appropriately modelled using networks with fuzzy values. Thus fuzzy location problems on networks arise; this paper deals with their general formulation and the description of the ways to solve them. Namely, we show the variety of problems that can be considered in this context and, for some of them, we propose the most operative approaches for their solution.
\end{abstract}

(C) 2003 Elsevier B.V. All rights reserved.

Keywords: Location; Fuzzy sets; Networks

\section{Introduction}

A location problem deals with the choice of a set of points for establishing certain facilities in such a way that, taking into account different criteria and verifying a given set of constraints, they optimally fulfill the needs of the users.

Some of the main location problems are modelled on a network or a graph. Thus, the vertices of the network represent the points where the users that demand the facility are, and the edges reveal the existence of a certain link between the vertices (for instance, roads joining cities). In general, the graphs represent deterministic situations where the points of demand and the links joining them are known.

\footnotetext{
* Corresponding author. Tel.: +34-922-318186; fax: +34-922-318170.

E-mail addresses: jamoreno@ull.es (J.A. Moreno Pérez),jmmoreno@ull.es (J.M. Moreno Vega), verdegay@ugr.es (J.L. Verdegay).
} 
Albeit from a theoretical point of view, we can say that the application contexts of all the location problems are identical (their defining elements are the same: a set of nodes, a set of edges and a distance matrix), though it is not clear whether these considerations are too simplistic. So, despite its formal correctness and the wide set of different models that can be considered, there is a wide range of real situations that, included perfectly in the problems that have been considered in this area, have not been studied in the specialized literature.

We specifically refer to the different possibilities that, with regard to the addressing of the problem, can arise when, besides the basic characteristics defining the problem, one adds a new feature which could be referred to as "the environment". To clarify this point, let us briefly think of the concept of vertex associated to a certain road map. Let us assume that we have a road map at hand, and that we are looking for a determined route. It is clear that in such a case, not all the points present on the map will have the same importance with regard to our interests; that is, it is patent that although all the vertices on the map may represent towns, not all of them will have the feature of provincial capital or village. From this point of view, it is evident that all the points on the map, and even all of those which frequently do not appear because of different reasons, may be considered as towns. However, not all those points will verify the property of being a town in the same way. The difference between a town with 25,000 inhabitants and another with 2500 inhabitants is obvious, but it is also obvious that both towns are settlements that verify the property "being a town" to a degree which in each case may be different. The possibility of including, in this simple case, all the possible settlements we are interested in, is usually made in a very strict way: we include all those nodes verifying exactly a given criterion (for instance number of inhabitants greater than 1000), and we discard from the map all those which do not verify that criterion. The concept of fuzzy set, and all the corresponding theory based on it, serves to bridge this misfunctioning since it allows us to introduce and handle sets for which the verification of the property defining them is measured in a graded manner, $[0,1]$-valued, and not exclusively in terms of 0,1 . In this context of graphs it makes sense, therefore, to consider fuzzy sets of vertices, so that each vertex verifies the assumed property to a certain degree. This same argument can be followed in reference to the edges of the graph. We can take, for example, the fact that the concept of road is basically a qualitative concept and, therefore, both a highway or a forest track are roads, each of them accomplishing the property of being a road to a different degree. Consequently, it also makes sense to consider fuzzy sets of edges, and much more generally, in spite of this set being fuzzy or not, and in spite of having a fuzzy set of vertices or not, to consider that the weights associated to the edges of the graph are defined by means of fuzzy numbers; that is, to consider that a fuzzy weight function is defined on the edges. It is patent that, except for very concrete cases, phrases like "it is not far, but it takes too long", "the road is very bad, but the trip is worth while", etc., are very frequent in day-to-day life. However, and in spite of the (vague) reality that they express, they are usually theoretically modelled in such a way that the vague concepts involved are modelled as numerical ones, when it seems much more convenient, in order not to lose the former vague nature of the problem, to model them properly establishing that imprecise nature they have.

A preliminary description of these aforementioned possibilities, in relation to location problems in graphs, is the essence of this work which, therefore, is structured as follows. In the next section, the most important concepts on location problems are introduced and the more relevant location models on fuzzy graphs are described. Then, in Section 3, with regard to exploring operative methods for solving the above models, some basic concepts on fuzzy graphs are presented. The solution 
methodologies here proposed are also enumerated and described, and some of them are exemplified by using the well-known median problem. Finally, Section 4 is devoted to pointing out the main conclusions and future research lines.

\section{Fuzzy location on networks: some models}

Let $G=(V, E)$ be a not-oriented, simple and connected graph with vertex set $V=\left\{v_{1}, v_{2}, \ldots, v_{n}\right\}$ and edge set $E=\left\{e_{1}, e_{2}, \ldots, e_{m}\right\}$. Each edge $e \in E$ is a continuous infinite and linear set of points joining two vertices $u, v \in V$, named extremes of $e$ that is represented by $e \in[u, v]$. Associated with each edge $e \in E$, there is a positive number $l(e)$ that can represent the length of the edge, the time needed to cover it, etc. In addition, associated to each vertex $v \in V$ there is a weight $w(v) \geqslant 0$ that is interpreted as the importance of that vertex. Let $w(\cdot)$ and $l(\cdot)$ be the corresponding weight and length functions defined on $V$ and $E$, respectively. The model is a network with four components: $N=(V, E, w, l)$.

A point $x$ on an edge $e \in[u, v]$ is determined by a value $\theta, 0 \leqslant \theta \leqslant 1$, that represents the portion of the edge between the extreme $u$ and the point $x$. We will denote this point by $x^{e}(\theta)$. Following this notation, the ends of the edge $e$ are $u=x^{e}(0)$ and $v=x^{e}(1)$. The interior points are given by $I(e)=\left\{x=x^{e}(\theta): 0<\theta<1\right\}$.

The two most representative problems in location theory on networks are the median and center problems. In both of them, one must find the point of the network $N$ that minimizes a function of the weighted distance to the vertices of $N$. In the median problem, the function to be minimized is the sum of the distances; in the center problem we want to minimize the maximum of the distances. Therefore, the median problem and the center problem are, respectively, formulated by

$$
\min _{x \in N} \sum_{v \in V} d(x, v) w(v) \text { and } \min _{x \in N} \max _{v \in V} d(x, v) w(v) .
$$

The first step in the solution of a location problem where all the points are possible solutions is to find a finite dominant set for the problem, i.e., a finite set that includes, at least, an optimal solution to the problem (see [9]). From the seminal paper of Hakimi [6], dominant finite sets are known for the median, consisting of the vertices, and for the center problem, that consists of the vertices and local centers, i.e., interior points in equilibrium between two vertices. Given the matrix of distances, the median is obtained in time $\mathrm{O}\left(n^{2}\right)$ and the center in time $\mathrm{O}(n m \log n)$. For the algorithmic resolution of these problems, the calculation of the matrix of distances needs the greatest computational effort; that means $\mathrm{O}(n m \log n)$ operations (see [6]).

In order to consider a fuzzy context in these problems, we will need the concept of fuzzy graph. Although the first definition of a fuzzy graph is due to Zadeh [15], it is generally assumed that this concept was originally proposed by Rosenfeld [13]. Subsequently, a lot of papers related to this concept have appeared and, namely, in [3] there is a summary of the main notions related with this topic. Also in [11], a state-of-the-art can be found. In particular, we are here interested in the median problem in a fuzzy context. This problem has been already described in part by Canos et al. $[1,2]$. However, the point of view adopted in this work will be much more general.

Namely, given a reference graph, $G=(V, E)$, we have a fuzzy network $N$, if there are appropriate defined membership functions for all or some of the following elements of $N$ : the vertex set $V$, the 
edge set $E$, the weights $w$ of the vertices or the lengths $l$ of the edges. Therefore, for the formulation of the fuzzy location problems on a graph or network we can consider the following four alternatives for the fuzzification of the model where only one of the elements in the model (vertices $V$, edges $E$, weights $w$ or lengths $l$ ) is given using fuzzy techniques.

(1) Location problems with fuzzy vertices: They appropriately models situations where the demand vertices verify a certain condition (for instance, being a relevant population) within a given graph. Those problems on a graph, for which the set of demand vertices $V$ is fuzzy, belong to this kind of model.

(2) Location problems with fuzzy edges: They address a parallel version to the above model, suitable when the edges $E$ are present in the models with a given degree and are modelled by a fuzzy set of pairs of vertices.

(3) Location problems with fuzzy weights: In a lot of situations, the information available does not allow us to assign deterministic values to the weights that represent the importance of the vertices. In those cases, in which, for instance, sentences appear like the weight of the vertex $v$ is about $w$, it is useful to consider that the weights in $w(v)$ are fuzzy numbers.

(4) Location problems with fuzzy lengths: We now deal with location problems on a network $N=(V, E, w, \tilde{l})$ where the lengths of the edges are not well known, and they are given by a function $\tilde{l}$ of fuzzy values, $\tilde{l}(e)$.

Obviously, the aforementioned classes can be combined giving rise to problems that belong simultaneously to two or more classes. So, we can consider location problems on a graph with both fuzzy vertices and edges, problems with both fuzzy weights and lengths, and so on.

\section{Solution methodologies}

Below, we deal with the methodologies that are appropriate to solve the problems corresponding to the models that are given in the above section. One way to solve these kinds of models is using the $\alpha$-cuts representation. The procedure consists of solving a finite series of crisp problems associated with the $\alpha$-cuts.

In order to consider the concepts $\alpha$-cuts on these problems we need some considerations. As the fuzziness can affect simultaneously to a variety of the elements of the model (weights, edges, vertices, length,...), and the universe of discourse for each of these elements is necessary different, the fuzzy element with respect to which we are considering the corresponding $\alpha$-level must be specified.

For the first two fuzzifications of the network model, the following concepts from the theory of fuzzy graphs will be useful. Consider the definition of fuzzy graph in [13].

Definition 1. A fuzzy graph ( $f$-graph) is the structure $G=(V, E, \sigma, \mu)$, where $V$ is the vertex set, $\sigma: V \rightarrow[0,1]$ is the membership function of the vertex set, $E$ is the edge set and $\mu: E \rightarrow[0,1]$ is the membership function of the edge set, where it verifies that

$$
\forall u, v \in V: \max _{e \in[u, v]} \mu(e) \leqslant \min \{\sigma(u), \sigma(v)\} .
$$


Definition 2. The $\alpha$-cut, $\alpha \in[0,1]$, of an $f$-graph $G=(V, E, \sigma, \mu)$ is the classical graph $G^{\alpha}=\left(V^{\alpha}, E^{\alpha}\right)$ with

$$
V^{\alpha}=\{v \in V: \sigma(v) \geqslant \alpha\} \quad \text { and } \quad E^{\alpha}=\{e \in E: \mu(e) \geqslant \alpha\} .
$$

Definition 3. A path $P$ between vertices $u$ and $v$ in an $f$-graph $G=(V, E, \sigma, \mu)$ is a sequence $e_{1}, e_{2}, \ldots, e_{k} \in E$ of edges that verifies:

$$
\begin{aligned}
& x^{e_{1}}(0)=u \quad \text { and } \quad x^{e_{k}}(1)=v, \\
& x^{e_{i}}(1)=x^{e_{i+1}}(0) \quad \forall i=1, \ldots, k-1 .
\end{aligned}
$$

Thus, the strength of a path $P$ in graph $G$ is the minimum of the membership functions of its edges, that is

$$
\mu(P)=\min _{e_{i} \in P} \mu\left(e_{i}\right)
$$

For every two vertices $u$ and $v$, let $P(u, v)$ denote the set of paths between $u$ and $v$.

Definition 4. Let $G=(V, E, \sigma, \mu)$ be an $f$-graph and $u, v \in V$. The connectedness level between $u$ and $v$ in $G$ is the value

$$
C(u, v)=\max _{P \in P(u, v)} \mu(P) .
$$

If $u=v$ then $C(u, v)=\infty$.

Definition 5. Let $G=(V, E, \sigma, \mu)$ be an $f$-graph. The connectedness level of $G$ is the value

$$
C(G)=\min _{u, v \in V} C(u, v) \text {. }
$$

This is an extension of the strength defined in [4,10].

For instance, in the $f$-graph shown in Fig. 1, the computations for the connectedness between vertices $v_{1}$ and $v_{4}$ are in Table 1 .

Since the location problems on networks have to be defined on a connected graph, one needs to be sure that the corresponding $\alpha$-cuts provide connected graphs. In order to formalize this idea, we use the notion of connectedness level $C(G)$ of the graph $G$. From all of the problems that appears in this framework, in the following section we will deal with those that provide the most concrete solution methodologies.

\subsection{Location problems with fuzzy vertices or edges}

One way to solve these kinds of models consists of solving the finite series of crisp problems associated with the $\alpha$-cuts for $\alpha \in(0, C(G)]$. The solution procedure (see [3,5]) consists of solving the location problem in $G^{\alpha_{i}}$, for the corresponding values $\alpha_{i}$ that give the intervals where the corresponding crisp models are constant. It is convenient to solve the crisp problems for the series of 


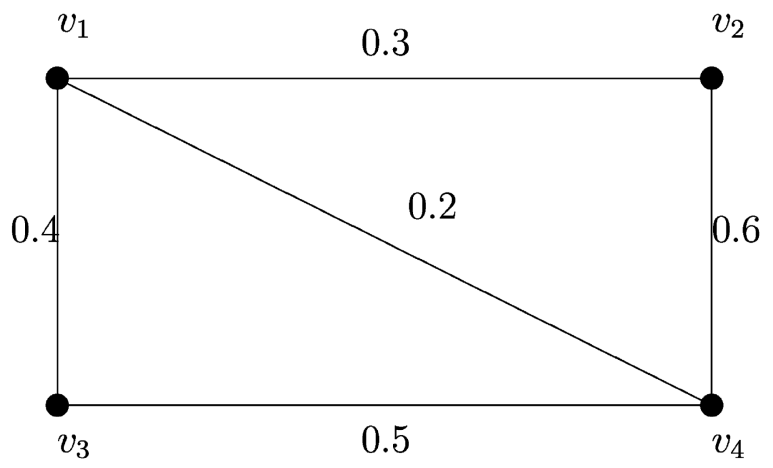

Fig. 1. An $f$-graph.

Table 1

Connectedness in an $f$-graph

\begin{tabular}{llll}
\hline$[u, v]$ & $l(u, v)$ & $\mu(u, v)$ & $P\left(v_{1}, v_{4}\right)$ \\
\hline$e_{1}=\left[v_{1}, v_{2}\right]$ & $(1.5,2,2.5)$ & 0.3 & $P_{1}=\left(e_{5}\right)$ \\
$e_{2}=\left[v_{2}, v_{4}\right]$ & $(1.5,2,3)$ & 0.6 & $P_{2}=\left(e_{1}, e_{2}\right)$ \\
$e_{3}=\left[v_{1}, v_{3}\right]$ & $(1,2,3)$ & 0.4 & $P_{3}=\left(e_{3}, e_{4}\right)$ \\
$e_{4}=\left[v_{3}, v_{4}\right]$ & $(0.5,1,1.5)$ & 0.5 & $C(u, v)=0.4$ \\
$e_{5}=\left[v_{1}, v_{4}\right]$ & $(3,4,5)$ & 0.2 & $C(G)=0.4$ \\
\hline
\end{tabular}

decreasing values $\alpha_{1}, \ldots, \alpha_{k}$ because the vertices are successively included in the computation of the corresponding minima. For solving the location problems, we must use the algorithms that are easily adapted to the updating of the distances when new edges appear. This is so important because the greatest computational effort in the solution of these location problems stems from the computation of the matrix distance.

\subsection{Location problems with fuzzy weights and lengths}

Since, in these problems, the objective function provides a fuzzy value for every feasible solution, the methodologies to solve them consist of applying the well-known comparing functions for fuzzy numbers (see [7]) to determine the location with minimum fuzzy objective function.

For instance, consider the problem of the median in a crisp graph with fuzzy weights. If we assume that the weights are triangular fuzzy numbers $w(v)=\left(w_{l}(v), w_{m}(v), w_{r}(v)\right)$, the membership function of the objective is obtained directly from Proposition 2 in [8]. So if we use the third index of Yager, the optimal location for the median can be obtained by solving the problem

$$
\min _{x \in N} \sum_{v \in V} d(x, v) \cdot \frac{1}{4}\left(w_{l}(v)+2 w_{m}(v)+w_{r}(v)\right) .
$$

Note that (1) corresponds to the problem of the median in the graph $G=(V, A)$ with weights $1 / 4\left(w_{l}(v)+2 w_{m}(v)+w_{r}(v)\right)$ for the vertices. An analogous result can be stated for the first index of Yager and similar properties can be obtained for other linear ordering functions. 
If the model includes fuzzy lengths, we need to analyze the properties of the fuzzy distance between points of the graph so as to be an optimal location for the facility. In the following, we assume that the linear ordering function is given and will be denoted by $f$.

\subsubsection{Finite dominating set for the median}

As we said above, the first step to solve these problems is to find a finite dominant set. We show how to get the finite dominant set for the median problem in a network with fuzzy lengths. A network with fuzzy lengths $N=(V, E, w, \tilde{l})$ consists of a network with vertex set $V=\left\{v_{1}, v_{2}, \ldots, v_{n}\right\}$, edge set $E=\left\{e_{1}, e_{2}, \ldots, e_{m}\right\}$ and crisp vertex weights $w$ where the length of every edge $e_{i}$ is given by a fuzzy number $\tilde{l}\left(e_{i}\right)$.

The operation of the insertion of a point in a network allows the extension of concepts about vertices to points.

Definition 6. Given a network with fuzzy lengths $N=(V, E, w, \tilde{l})$ and a point $x=x^{e}(\theta)$ on $e \in[u, v]$, the insertion of $x$ in $N$ is an operation that provides a new graph $N_{\cdot x}=\left(V \cdot x, E_{\cdot x}, w_{\cdot x}, \tilde{l}_{\cdot x}\right)$ where the new vertex set is $V_{\cdot x}=V \cup\{x\}$ with new weights given by $w_{\cdot x}(x)=0$ and $w_{\cdot x}(v)=w(v) \forall v \in V$; and the new edge set is

$$
E_{\cdot x}=(E-\{e\}) \cup\left\{e_{1}, e_{2}\right\}
$$

where the two new edges are $[u, x]=\left\{e_{1}\right\}$ and $[x, v]=\left\{e_{2}\right\}$, and the new lengths given by

$$
\begin{aligned}
& \tilde{l}_{\cdot x}\left(e_{1}\right)=\theta \tilde{l}(e), \\
& \tilde{l}_{\cdot x}\left(e_{2}\right)=(1-\theta) \tilde{l}(e), \\
& \tilde{l}_{\cdot x}\left(e^{\prime}\right)=\tilde{l}\left(e^{\prime}\right) \quad \forall e^{\prime} \in E \cap E_{\cdot x} .
\end{aligned}
$$

Note that $\tilde{l}_{x}(e)=\infty$ is another way to exclude $e$ from the graph. The insertion of points in the network with fuzzy length allows us to extend the concepts about vertices to be applied to points on the network (see Fig. 2 and Table 2).

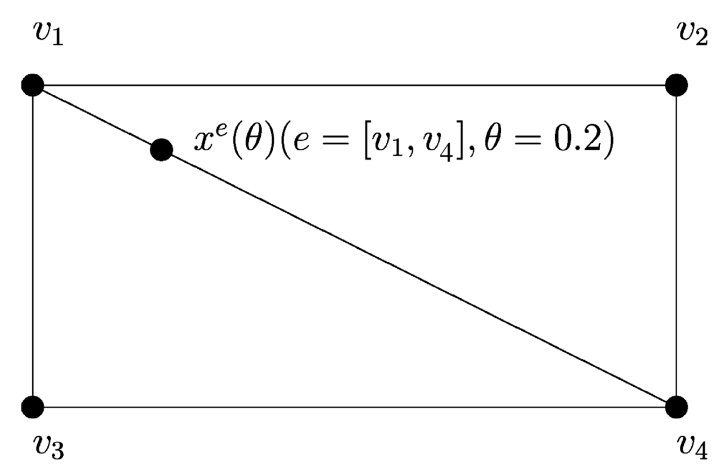

Fig. 2. Insertion of a point in a network with fuzzy lengths. 
Table 2

Lengths of the new graph

\begin{tabular}{llll}
\hline$[u, v]$ & $l(u, v)$ & {$[u, v]$} & $l \cdot x(u, v)$ \\
\hline$e_{1}=\left[v_{1}, v_{2}\right]$ & $(1.5,2,2.5)$ & $e_{1}=\left[v_{1}, v_{2}\right]$ & $(1.5,2,2.5)$ \\
$e_{2}=\left[v_{2}, v_{4}\right]$ & $(1.5,2,3)$ & $e_{2}=\left[v_{2}, v_{4}\right]$ & $(1.5,2,3)$ \\
$e_{3}=\left[v_{1}, v_{3}\right]$ & $(1,2,3)$ & $e_{3}=\left[v_{1}, v_{3}\right]$ & $(1,2,3)$ \\
$e_{4}=\left[v_{3}, v_{4}\right]$ & $(0.5,1,1.5)$ & $e_{4}=\left[v_{3}, v_{4}\right]$ & $(0.5,1,1.5)$ \\
$e_{5}=\left[v_{1}, v_{4}\right]$ & $(3,4,5)$ & $e_{6}=\left[v_{1}, x^{e}(\theta)\right]$ & $(0.6,0.8,1.0)$ \\
& & $e_{7}=\left[x^{e}(\theta), v_{4}\right]$ & $(2.4,3.2,4.0)$ \\
\hline
\end{tabular}

Let $P$ be a path between vertices $u$ and $v$ in network $N=(V, E, w, \tilde{l})$. The length of the path $P$ is

$$
\tilde{l}(P)=\sum_{e_{i} \in P} \tilde{l}\left(e_{i}\right)
$$

To avoid dispersion difficulties, and with not formal restrictions, we consider this fuzzy number has left and right margins equal to the respective maximum and minimum of the corresponding fuzzy numbers. Then, let $P\left(v_{i}, v_{j}\right)$ be the set of all the paths between the vertices $v_{i}$ and $v_{j}$, and consider the linear ordering function $f$ for fuzzy numbers that have been selected. Then the shortest path between two vertices is defined as follows.

Definition 7. A path $P^{*} \in P\left(v_{i}, v_{j}\right)$ is the shortest path between $v_{i}$ and $v_{j}$ if and only if

$$
\tilde{l}\left(P^{*}\right)=\min \left\{\tilde{l}(P) ; P \in P\left(v_{i}, v_{j}\right)\right\} .
$$

Note that the computation of this value must be done through the ordering function $f$ that has been previously selected. Thus, depending on this function, different results can be obtained because different shortest paths will be obtained. From now on $P_{\min }\left(v_{i}, v_{j}\right)$ will denote the set of shortest paths between $v_{i}$ and $v_{j}$.

Definition 8. The distance, $\tilde{d}\left(v_{i}, v_{j}\right)$, between the vertices $v_{i}$ and $v_{j}$ is the length of any shortest path between $v_{i}$ and $v_{j}$. That is

$$
\tilde{d}\left(v_{i}, v_{j}\right)=\tilde{l}(P) \quad \forall P \in P_{\min }\left(v_{i}, v_{j}\right) .
$$

The distance between two points on the network $N=(V, E, w, \tilde{l})$ is defined as follows.

Definition 9. The distance, $\tilde{d}(x, y)$, between the points $x$ and $y$ on the graph $N=(V, E, w, \tilde{l})$ is the distance between the vertices $x$ and $y$ of the graph

$$
\left(N_{\cdot x}\right)_{\cdot y}=\left(\left(V_{\cdot x}\right)_{y},\left(E_{\cdot x}\right)_{y},\left(w_{\cdot x}\right) \cdot y,\left(l_{\cdot x}\right) \cdot y\right)
$$




\subsubsection{Properties of the distance}

Given an edge $e \in[u, v]$ and a point $z$, non-interior to $e$, the distance from $x=x^{e}(\theta)(0 \leqslant \theta \leqslant 1)$ to $z$ is

$$
\tilde{d}\left(x^{e}(\theta), z\right)=\min \{\tilde{d}(u, z)+\theta \tilde{l}(e), \tilde{d}(v, z)+(1-\theta) \tilde{l}(e)\} .
$$

From these definitions we have the following properties.

Proposition 10. The distance between the points of the edge $e \in[u, v]$ and the non-interior point $z$ is

$$
\tilde{d}\left(x^{e}(\theta), z\right)= \begin{cases}\tilde{d}(u, z)+\theta \tilde{l}(e), & 0 \leqslant \theta \leqslant \theta_{z}^{e}, \\ \tilde{d}(v, z)+(1-\theta) \tilde{l}(e), & \theta_{z}^{e} \leqslant \theta \leqslant 1,\end{cases}
$$

where

$$
\theta_{z}^{e}=\frac{f(\tilde{d}(v, z)+\tilde{l}(e)-\tilde{d}(u, z))}{2 f(\tilde{l}(e))} .
$$

Proof. The distance between $x^{e}(\theta)$ and the point $z$ is given by formula (2). So, this distance will be $\tilde{d}(u, z)+\theta \tilde{l}(e)$ while

$$
\tilde{d}(u, z)+\theta \tilde{l}(e) \leqslant \tilde{d}(v, z)+(1-\theta) \tilde{l}(e) .
$$

This last condition is true when

$$
f(\tilde{d}(u, z)+\theta \tilde{l}(e)) \leqslant f(\tilde{d}(v, z)+(1-\theta) \tilde{l}(e)) .
$$

That is, if $\theta \leqslant \theta_{z}^{e}$. Note that $0 \leqslant \theta_{z}^{e} \leqslant 1$.

Proposition 11. Let a point $z$ non-interior to edge $e \in[u, v]$ and $\theta_{z}^{e}$ given by formula (3). One verifies

(1) if $\theta_{z}^{e}=0$ then the function $\tilde{d}\left(x^{e}(\cdot), z\right)$ is linear and decreasing on $[0,1]$ with slope $-\tilde{l}(e)$,

(2) if $\theta_{z}^{e}=1$ then the function $\tilde{d}\left(x^{e}(\cdot), z\right)$ is linear and increasing on $[0,1]$ with slope $\tilde{l}(e)$,

(3) if $0<\theta_{z}^{e}<1$ then the function $\tilde{d}\left(x^{e}(\cdot), z\right)$ is linear and increasing on $\left[0, \theta_{z}^{e}\right]$ with slope $\tilde{l}(e)$, and linear and decreasing on $\left[\theta_{z}^{e}, 1\right]$ with slope $-\tilde{l}(e)$.

We use the definition of a concave fuzzy function given in [14].

Definition 12. A fuzzy function $F$ is concave in $\Theta$ if and only if,

$$
F\left(\lambda \theta_{1}+(1-\lambda) \theta_{2}\right) \geqslant \lambda F\left(\theta_{1}\right)+(1-\lambda) F\left(\theta_{2}\right) \quad \forall \theta_{1}, \theta_{2} \in \Theta .
$$

Proposition 13. The function $\tilde{d}\left(x^{e}(\cdot), z\right)$ is concave in $[0,1]$.

Proof. We show that $\forall \theta_{1}, \theta_{2} \in[0,1]$, one verifies

$$
F\left(\lambda \theta_{1}+(1-\lambda) \theta_{2}\right) \geqslant \lambda F\left(\theta_{1}\right)+(1-\lambda) F\left(\theta_{2}\right)
$$

in all the possible cases for $\theta_{z}^{e}$, i.e., $\theta_{z}^{e}=0, \theta_{z}^{e}=1$ and $0<\theta_{z}^{e}<1$. 
(1) If $\theta_{z}^{e}=0$, then

$$
\tilde{d}\left(x^{e}\left(\lambda \theta_{1}+(1-\lambda) \theta_{2}\right), z\right)=\tilde{d}(v, z)+\left(1-\left(\lambda \theta_{1}+(1-\lambda) \theta_{2}\right)\right) \tilde{l}(e)
$$

and

$$
\begin{aligned}
& \lambda \tilde{d}\left(x^{e}\left(\theta_{1}\right), z\right)+(1-\lambda) \tilde{d}\left(x^{e}\left(\theta_{2}\right), z\right) \\
& \quad=\tilde{d}(v, z)+\left(\lambda\left(1-\theta_{1}\right)+(1-\lambda)\left(1-\theta_{2}\right)\right) \tilde{l}(e) .
\end{aligned}
$$

Using the ordering function $f$ for fuzzy numbers and with some algebra with real numbers it follows that expressions (4) and (5) are the same.

(2) The proof for $\theta_{z}^{e}=1$ is similar to the last case.

(3) If $0<\theta_{z}^{e}<1$, we consider three subcases:

(a) $\theta_{1} \leqslant \theta_{z}^{e}$ and $\theta_{2} \leqslant \theta_{z}^{e}$. The situation is like in the case $\theta_{z}^{e}=1$.

(b) $\theta_{1} \geqslant \theta_{z}^{e}$ and $\theta_{2} \geqslant \theta_{z}^{e}$. The situation is like in the case $\theta_{z}^{e}=0$.

(c) $\theta_{1} \leqslant \theta_{z}^{e}$ and $\theta_{2} \geqslant \theta_{z}^{e}$. Assume that $\lambda \theta_{1}+(1-\lambda) \theta_{2} \leqslant \theta_{z}^{e}$ (if $\lambda \theta_{1}+(1-\lambda) \theta_{2} \geqslant \theta_{z}^{e}$ it is the mirror case). Like above, using the ordering function $f$ for fuzzy numbers and with some algebra we obtain that

$$
\tilde{d}\left(x^{e}\left(\lambda \theta_{1}+(1-\lambda) \theta_{2}\right), z\right) \geqslant \lambda \tilde{d}\left(x^{e}\left(\theta_{1}\right), z\right)+(1-\lambda) \tilde{d}\left(x^{e}\left(\theta_{2}\right), z\right)
$$

if and only if

$$
\theta_{2} \geqslant \frac{f(\tilde{d}(v, z)+\tilde{l}(e)-\tilde{d}(u, z))}{2 f(\tilde{l}(e))}=\theta_{z}^{e}
$$

that is true.

Using the above properties, we obtain the following result that provides a finite dominant set for the median problem with fuzzy lengths. Based on this result we will provide a solution method.

Theorem 14. Let $N=(V, E, w, \tilde{l})$ be a network with fuzzy lengths. There is at least one vertex in $V$ that is a median of $N$.

Proof. Since the multiplication by a positive scalar of a concave function is also a concave function, the sum of concave functions is also a concave one and, in addition, the minimum of any concave function in an interval is reached at one of its extremes, the proof easily follows.

This justifies the usual rule applied by the taxi drivers that always want the taxi stops at the corner of the streets.

\subsection{An example}

Let us assume that we are concerned with the necessity of suggesting the places in which taxi stops must be located in a certain town. The geographical areas in which these taxi stops could be located is vaguely known, but the exact place where finally they will be is unknown. Edges (streets) will have associated on the one hand fuzzy weights because the weights represent the time of traversing 


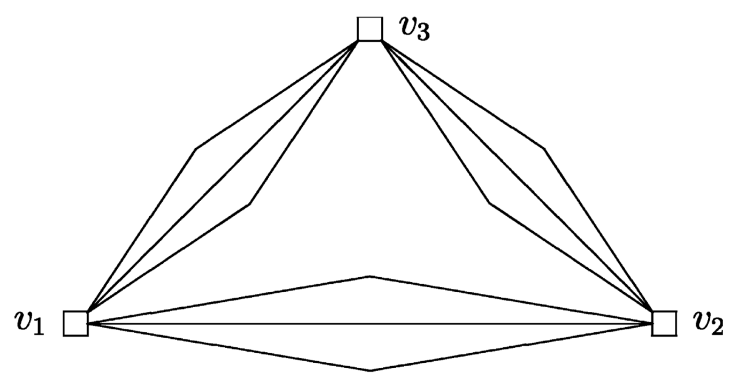

Fig. 3. Graph $G$ for the example.

the streets, and on the other they will have some membership degree giving the accomplishment level of a prescribed property, as for instance may be "good street". Under these conditions, in order to illustrate the methodology developed here, let us consider the following example.

Let $G$ be the graph consisting of three vertices $\left\{v_{1}, v_{2}, v_{3}\right\}$ that represents three towns and three edges joining each pair of vertices that represents three alternative paths between them (see Fig. 3 ). The three edges joining vertices $v_{i}$ and $v_{j}$ are denoted by $e_{i j}^{k}$ for $k=1,2,3$. We have vague information on the length of the roads and on their quality.

For each edge, we have the fuzzy length and the fuzzy membership value for some quality condition, for instance to be a good road. We want to get the median of the corresponding fuzzy model. We can consider three situations:

(1) The length of each road is given but the property to be a good road is evaluated by a membership function. This is a model with fuzzy set of edges.

(2) The length of each edge is represented by a fuzzy number, but we consider that all the roads are good enough, i.e., the membership function is 1 for all of them.

(3) The length of each road is given by a fuzzy number and set of good roads is a fuzzy set.

To solve the last case we get in Table 3, for each possible path, the fuzzy length, the membership for the set of good roads and the corresponding value for the third Yager index.

The connectedness level is 0.75 . The values for $\alpha$ corresponding to the different crisp graphs are $[0,0.25],(0.25,0.5]$ and $(0.5,0.75]$. A possible methodology is to solve the median problem for these cuts. Note that they are not crisp median problems because for each cut, we have a crisp graph with fuzzy lengths. These problems are solved using the ordering function corresponding to the third Yager index.

Then, if $\alpha \in[0,0.25]$ then the median is $v_{1}$, if $\alpha \in(0.25,0.5]$ then it is $v_{2}$ and for $\alpha \in(0.5,0.75]$ the edge $\left[v_{1}, v_{2}\right]$ is the set of medians.

\section{Conclusions and future research}

In this work, we have considered some location problems on fuzzy graphs that appropriately model several contexts that appear in real situations. Furthermore, we have proposed suitable methodologies 
Table 3

\begin{tabular}{|c|c|c|c|c|c|c|c|c|}
\hline & \multicolumn{4}{|c|}{ Vertex 2} & \multicolumn{4}{|c|}{ Vertex 3} \\
\hline & Path & Length & Strength & Index & Path & Length & Strength & Index \\
\hline & $e_{12}^{1}$ & $(1.5,2,2.5)$ & 0.25 & 2.000 & $e_{13}^{1}$ & $(1,2,3)$ & 0.25 & 2.000 \\
\hline & $e_{12}^{2}$ & $(3,4,5)$ & 0.50 & 4.000 & $e_{13}^{2}$ & $(4.5,5,6)$ & 0.5 & 5.125 \\
\hline $\mathrm{v}$ & $e_{12}^{3}$ & $(4,6,8)$ & 0.75 & 6.000 & $e_{13}^{3}$ & $(6,7,8)$ & 0.75 & 7.000 \\
\hline $\mathrm{e}$ & $e_{13}^{1}, e_{32}^{1}$ & $(3,5,6.5)$ & 0.25 & 4.875 & $e_{12}^{1}, e_{23}^{1}$ & $(3.5,5,6)$ & 0.25 & 4.875 \\
\hline $\mathrm{r}$ & $e_{13}^{1}, e_{32}^{2}$ & $(4.5,6,8)$ & 0.25 & 6.125 & $e_{12}^{1}, e_{23}^{2}$ & $(5,6,7)$ & 0.25 & 6.000 \\
\hline $\mathrm{t}$ & $e_{13}^{1}, e_{32}^{3}$ & $(7,9,11)$ & 0.25 & 9.000 & $e_{12}^{1}, e_{23}^{3}$ & $(7.5,9,10.5)$ & 0.25 & 9.000 \\
\hline e & $e_{13}^{2}, e_{32}^{1}$ & $(6.5,8,9.5)$ & 0.50 & 8.000 & $e_{12}^{2}, e_{23}^{1}$ & $(5,7,8.5)$ & 0.5 & 6.875 \\
\hline \multirow[t]{2}{*}{$\mathrm{x}$} & $e_{13}^{2}, e_{32}^{2}$ & $(8,9,11)$ & 0.25 & 9.250 & $e_{12}^{2}, e_{23}^{2}$ & $(6.5,8,10)$ & 0.25 & 8.625 \\
\hline & $e_{13}^{2}, e_{32}^{3}$ & $(10.5,12,14)$ & 0.50 & 12.125 & $e_{12}^{2}, e_{23}^{3}$ & $(9,11,14)$ & 0.5 & 11.250 \\
\hline \multirow[t]{5}{*}{1} & $e_{13}^{3}, e_{32}^{1}$ & $(8,10,11.5)$ & 0.50 & 9.875 & $e_{12}^{3}, e_{23}^{1}$ & $(6,9,11.5)$ & 0.5 & 8.875 \\
\hline & $e_{13}^{3}, e_{32}^{2}$ & $(9.5,11,13)$ & 0.25 & 11.125 & $e_{12}^{3}, e_{23}^{2}$ & $(7.5,10,13)$ & 0.25 & 10.125 \\
\hline & $e_{13}^{3}, e_{32}^{3}$ & $(12,14,16)$ & 0.75 & 14.000 & $e_{12}^{3}, e_{23}^{3}$ & $(10,13,16)$ & 0.75 & 13.000 \\
\hline & & & & & $e_{23}^{1}$ & $(2,3,3.5)$ & 0.5 & 2.875 \\
\hline & & & & & $e_{23}^{2}$ & $(3.5,4,5)$ & 0.25 & 4.125 \\
\hline $\mathrm{v}$ & & & & & $e_{23}^{3}$ & $(6,7,8)$ & 0.75 & 7.000 \\
\hline $\mathrm{e}$ & & & & & $e_{21}^{1}, e_{13}^{1}$ & $(2.5,4,5.5)$ & 0.25 & 4.000 \\
\hline $\mathrm{r}$ & & & & & $e_{21}^{1}, e_{13}^{2}$ & $(6,7,8.5)$ & 0.25 & 7.125 \\
\hline $\mathrm{t}$ & & & & & $e_{21}^{1}, e_{13}^{3}$ & $(7.5,9,10.5)$ & 0.25 & 9.000 \\
\hline $\mathrm{e}$ & & & & & $e_{21}^{2}, e_{13}^{1}$ & $(4,6,8)$ & 0.25 & 6.000 \\
\hline $\mathrm{x}$ & & & & & $e_{21}^{2}, e_{13}^{2}$ & $(7.5,9,11)$ & 0.5 & 9.125 \\
\hline & & & & & $e_{21}^{2}, e_{13}^{3}$ & $(9,11,13)$ & 0.5 & 11.000 \\
\hline 2 & & & & & $e_{21}^{3}, e_{13}^{1}$ & $(5,8,11)$ & 0.25 & 8.000 \\
\hline & & & & & $e_{21}^{3}, e_{13}^{2}$ & $(8.5,11,14)$ & 0.5 & 11.125 \\
\hline & & & & & $e_{21}^{3}, e_{13}^{3}$ & $(10,13,16)$ & 0.75 & 13.000 \\
\hline
\end{tabular}

for solving the four basic models considered. However, other methodologies could be considered, namely those obtained from the proposals in [12] to get the shortest paths in graphs with fuzzy lengths. We are now studying this alternative approach.

\section{Acknowledgements}

Research carried out under projects TIC2002-04242-CO3-01,02 (70\% of founds for project TIC2002-04242-CO3-01 corresponds to FEDER founds). The first author has been supported by the Consejería de Educación, Cultura y Deportes del Gobierno de Canarias through project PI1999/116. The second author has been supported by the Consejería de Educación, Cultura y Deportes del Gobierno de Canarias and by the University of La Laguna through projects PI1999/116 and 221/52/99, respectively. The third author has been supported by the Spanish MECD under project PB98-1305. 


\section{References}

[1] M.J. Canos, C. Ivorra, V. Liern, An exact algorithm for the fuzzy p-median problem, European J. Oper. Res. 116 (1999) 80-86.

[2] M.J. Canos, C. Ivorra, V. Liern, The fuzzy p-median problem: a global analysis of the solutions, European J. Oper. Res. 130 (2001) 430-436.

[3] S. Chanas, M. Delgado, J.L. Verdegay, M.A. Vila, Fuzzy optimal flow on imprecise structures, European J. Oper. Res. 83 (1995) 568-580.

[4] M. Delgado, J.L. Verdegay, M.A. Vila, On fuzzy tree definition, European J. Oper. Res. 22 (1985) $243-249$.

[5] M. Delgado, J.L. Verdegay, M.A. Vila, On valuation and problems in fuzzy graphs: a general approach and some particular cases, ORSA J. Comput. 2 (1990) 74-84.

[6] S.L. Hakimi, Optimal locations of switching centers and the absolute centers and medians of a graph, Oper. Res. 12 (1964) 450-459.

[7] F. Herrera, J.L. Verdegay, Three models of fuzzy integer linear programming, European J. Oper. Res. 83 (1995) 581-593.

[8] F. Herrera, J.L. Verdegay, Fuzzy boolean programming problems with fuzzy costs: a general study, Fuzzy Sets and Systems 81 (1996) 57-76.

[9] J.N. Hooker, R.S. Garfinkel, C.K. Chen, Finite dominating sets for network location problems, Oper. Res. 39 (1991) $100-118$.

[10] A. Kaufman, Introduction a la Theorie des Sous-emsembles Flous, vol. I, Masson et Cie, 1973.

[11] J.N. Mordeson, P.S. Nair, Fuzzy graphs and fuzzy hypergraphs, Studies in Fuzziness and Soft Computing, Physica-Verlag, Wurzburg, 2000.

[12] S. Okada, T. Soper, A shortest path problem on a network with fuzzy arc lengths, Fuzzy Sets and Systems 109 (2000) 129-140.

[13] A. Rosenfeld, Fuzzy graph, in: L.A. Zadeh, K.S. Fu, K. Tanaka, M. Shimura (Eds.), Fuzzy Sets and Their Applications to Cognitive and Decision Processes, Academic Press, New York, 1975, pp. 79-97.

[14] Y.R. Syau, On convex and concave fuzzy mappings, Fuzzy Sets and Systems 103 (1999) $163-168$.

[15] L.A. Zadeh, Fuzzy sets, Information and Control 8 (1965) 338-353. 